\section{ZOOM IN FOR EDUCATION, INNOVATION AND DEMONSTRATION}

Philips will be offering visitors to BDTA Dental Showcase the chance to gain CPD via an education lecture theatre on stand K16. Speakers will include Professor Damien Walmsley and Zaki Kanaan, who will also be demonstrating the latest tooth whitening technology and techniques.

In the last year Philips has upped its focus on professional education still further with the introduction of a dedicated Professional Education

Manager, a Transitional Support Programme for Dental Hygienists and Therapists, and programmes for dental students and Foundation Dentists.

Lighting up the Philips stand for the first time will be the Company's new Zoom WhiteSpeed, a light-activated tooth whitening system with a variable range of intensity settings which enables dentists to make adjustments for patients who may experience transient sensitivity during the whitening process. As a result dentists are able to extend the chairside experience to four whitening cycles and achieve better results for their patients. The new LED technology used in the system also provides a significantly longer-lasting light source (50,000 hours) and uses 41\% less energy than previous models.

Delegates will also be encouraged to try two of Philips' latest products for themselves in brushing booths on the stand.

For the first time a product from an aligned sector of the Philips' stable, Respironics, will be debuted at a dental show in the UK. The Respironics device is designed to tackle obstructive sleep apnoea, a common disorder affecting at least $4 \%$ of men and $2 \%$ of women.

Visit Philips on stand K16. You can also preregister for talks on the stand by calling 08000323005 or 08000567222.

\title{
WIN ALL-EXPENSES PAID TECHNOLOGY TRAINING
}

Ivoclar Vivadent are excited to be attending BDTA Dental Showcase as exhibitors, whilst also exclusively sponsoring the BDTA's technician focused 'Tech-Zone'.

Taking place on Saturday 6 October 2012, the 'Tech Zone' has been specifically developed by the BDTA to offer dental technicians a fantastic opportunity to discover what's new in the fast moving world of dental technology. With every session qualifying for CPD, delegates will gain direct access to some of the UK's leading technicians, including Rob Storrar and Bill Sharpling, as they provide live demonstrations and top tips and techniques throughout the day. As exclusive sponsors, Ivoclar Vivadent are offering two lucky 'Tech-Zone' delegates the chance to win a free place on one of their IPS e.max or Next Generation Denture Courses, at the ICDE Leicester, with all expenses paid.

To find out more about Ivoclar Vivadent, visit stand E09, www.ivoclar-vivadent.co.uk or call 0116284788.

\section{CLAIM A FREE IPAD3}

Claim a free iPad3 from Bien-Air on stand C07 when you purchase the multi-user iChiropro that incorporates the complete sequences of the leading implant manufacturers. Each system is supplied with a micro-Series contra-angle handpiece, a unique irrigation system for greater comfort and one of the smallest heads on the market. This physiodispenser is controlled from an iPad and the intuitive, ergonomic application ensures significant time savings. Available free from Apple, the iChiropro application has numerous customisation options.

Take advantage of free upgrade to Micro-Series for existing MC2 Isolite, MC3 and MX users. MicroSeries contra-angles offer you the best in terms of grip and balance, guaranteeing more precise control and less fatigue. Smaller than conventional contra-angles, they are ultra-precise, reliable and quiet.

The MCX LED Micromotor Kit is being offered at just $£ 995$ + VAT. No maintenance is required and it is the smallest and lightest brushless micromotor ever from Bien-Air.

Visit stand C07 for further information. 